\title{
Electronic Case-Report Forms of Symptoms and Impairments of Peripheral Neuropathy
}

\author{
Peter J. Dyck, David W. Turner, Jenny L. Davies, Peter C. O'Brien, \\ P. James B. Dyck, Cynthia A. Rask, and the rhNGF group
}

\begin{abstract}
Background and objective: For the conduct of controlled clinical trials, epidemiologic surveys or even of medical practice of varieties of peripheral neuropathy, the usefulness, error rate and cost-effectiveness of scannable case-report forms has not been studied. Materials and methods: The overall performance, the frequency of the problems identified and corrected, and the time saved from use of a standard paper case report form was evaluated in multicenter treatment trials, single center epidemiologic surveys and in our neurologic practice. The paper case report form (Clinical Neuropathy Assessment [CNA]) for pen entry at study medical centers for patient, disease and demographic information (Lower Limb Function [LLF] and Neuropathy Impairment Score [NIS]) can be faxed to a core Reading and Quality Assurance Center where the form and data is electronically and interactively evaluated and corrected, if needed, by participating medical centers before electronic entry into database. Observations and conclusions: 1) The approach provides a standard, scannable paper case report form for pen entry of neuropathy symptoms, impairments and disability at the bedside or in the office which is retained as a source document at the participating medical center but a facsimile can be transferred instantaneously, its data can be programmed, interactively evaluated, modified and stored while maintaining an audit trail; 2) it allowed efficient and accurate reading, transfer, analysis, and storage of data of more than 15,000 forms used in multicenter trials; 3 ) in 500 consecutive CNA evaluations, software programs identified and facilitated interactive corrections of omissions, discrepancies, and disease and study inconsistencies, introducing only a few readily identified and corrected entry errors; and 4) use of programmed, as compared to non-programmed assessment, was more accurate than double keyboard entry of data and was approximately five times faster.
\end{abstract}

RÉSUMÉ: Formulaires électroniques d'exposé de cas pour les symptômes et l'invalidité dans la neuropathie périphérique. Introduction et Objectif: Pour la conduite d'essais thérapeutiques, d'enquêtes épidémiologiques ou même en pratique médicale portant sur différentes neuropathies périphériques, l'utilité, le taux d'erreur et le coût/bénéfice des formulaires d'exposé de cas (FEC) n'ont pas été étudiés. Matériels et Méthodes: La performance globale, la fréquence de problèmes identifiés et corrigés, et le temps épargné par l'utilisation de FEC papier standards ont été évalués dans des essais multicentres, des enquêtes épidémiologiques réalisées dans un centre et dans notre pratique neurologique. Le FEC papier (Évaluation clinique de la neuropathie [ÉCN]) rempli à la main au site de l'étude et contenant l'information sur le patient, la maladie et les données démographiques (Lower Limb Function - LLF et Neuropathy Impairment Score - NIS) peut être transmis par télécopieur à un centre de lecture et de contrôle de qualité où le formulaire et les données sont évalués et corrigés électroniquement et interactivement au besoin par les centres médicaux participants avant la saisie électronique dans une base de données. Observations et Conclusions: 1) Cette approche fournit un FEC papier standard, complété à la main au lit du malade ou au bureau, pour saisie électronique sur les symptômes de neuropathie, les déficits et l'invalidité qui est conservé comme document source au site participant. Un fac-similé peut être transféré instantanément, ses données peuvent être programmées et évaluées interactivement, modifiées et conservées tout en maintenant un document source pour vérification; 2) elle permet la lecture, le transfert, l'analyse et la conservation efficace et précise de données de plus de 15000 formulaires utilisés dans des essais multicentres; 3) dans 500 ÉCNs consécutives, des programmes informatiques ont identifié et facilité la correction interactive d'omissions, de divergences et de contradictions quant à la maladie et à l'étude, introduisant seulement un petit nombre d'erreurs d'entrée de données facilement identifiées et corrigées; et 4) l'utilisation d'une évaluation programmée comparée à une évaluation non programmée était plus exacte qu'une double entrée de données au clavier et était approximativement cinq fois plus rapide.

Can. J. Neurol. Sci. 2002; 29: 258-266

For the conduct of controlled clinical trials, epidemiologic surveys and monitoring course of disease, use of scannable case report forms may be preferable, for some purposes, than previously available methods.

Here we describe a scannable case report form, Clinical
From the Peripheral Neuropathy Research Center (PJD, JLD, PJBD), Department of System Support Services (DWT), Section of Biostatistics (PCO), Mayo Clinic and Mayo Foundation, Rochester, MN; and FDA, Rockville, MD (CAR), USA ReCeIVEd DeCEMber 4, 2001. ACCEPTED IN FINAL FORM ApRIL 10, 2002. Reprint requests to: Peter J. Dyck, 200 First Street, SW; Rochester, MN 55905 USA 
Neuropathy Assessment (CNA), using Teleform technology (Cardiff, Inc., San Marcos, CA) (and the additional computer hardware and software approaches we developed) for the quantitative and serial clinical evaluation of patients with peripheral neuropathy. This scannable case report form was designed to: 1) record patient, disease, study, demographic and standard neuropathy test results of impairments, symptoms and disabilities on a scannable paper form which could be used to flexibly record all items of tests or only a subset (a limited region of the body, certain classes of neurons or a single nerve) of the tests; 2) provide automatic and interactive approaches so as to read (by scanner or fax), transfer (the facsimile of CNA and data), interactively evaluate by computer software (according to pre-determined criteria for disease and study), and store the data (in database); and 3) perform all of these functions quickly and accurately maintaining the needed audit trail.

Here, in addition to describing CNA, we analyze how it performed in multicenter controlled clinical trials, the frequency of the problems which occur and which were remedied with its use, the time that can be saved by using these automated approaches, the degree of accuracy and its special use in clinical practice.

\section{METHODS}

\section{Description of the scannable case report form (Clinical Neuropathy Assessment [CNA])}

Clinical Neuropathy Assessment is a pre-printed 12-page peripheral neuropathy case report form comprised of a title page

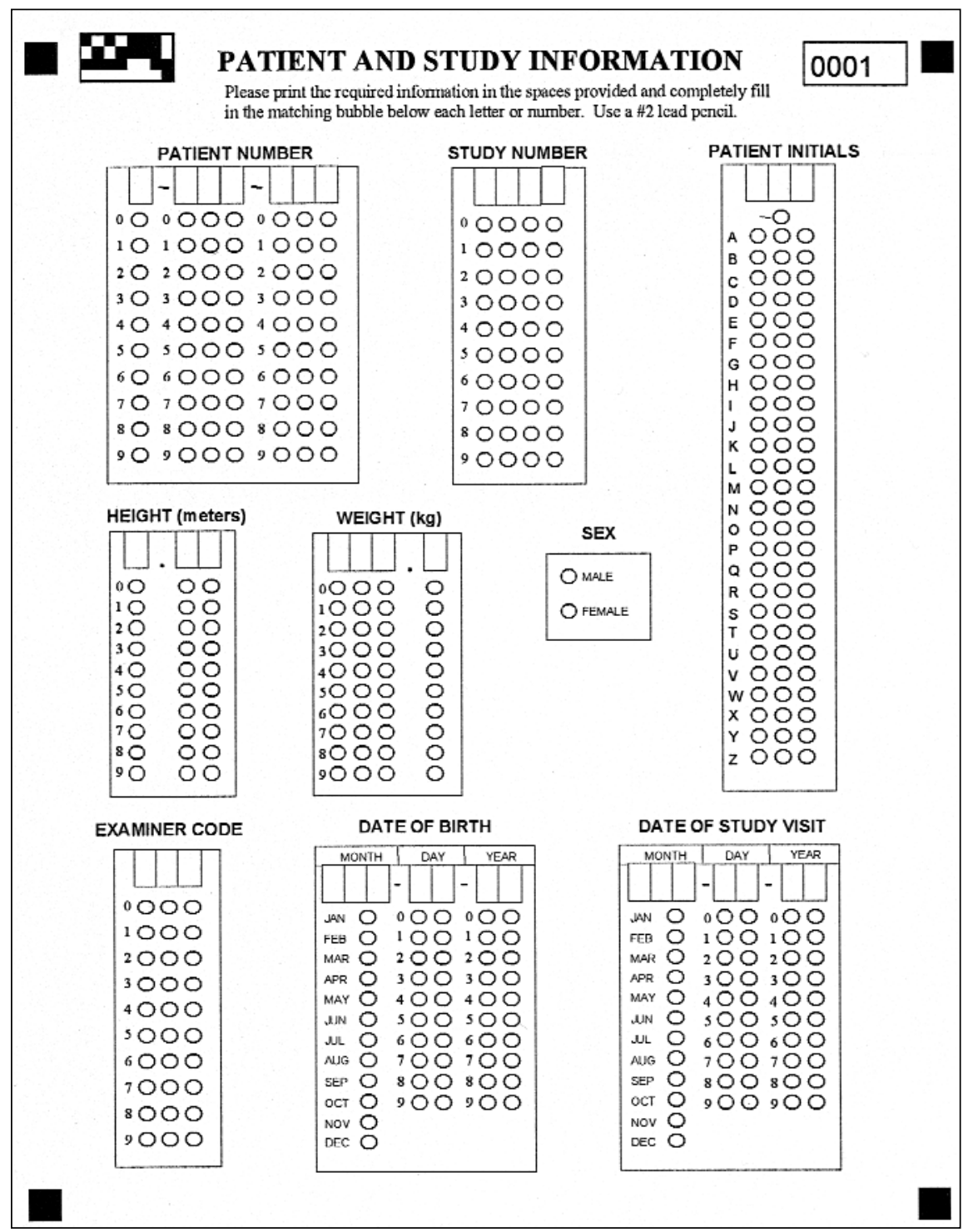

Figure 1: The patient and study information page (page 2) of CNA. As described in text, all pages of CNA have a unique number (in the right-upper box), corner symbols for proper paper alignment, and checkerboard symbols (left upper) to identify that the CNA belongs to a specific study (or group of studies). Note that instructions are provided on how the data is to be judged and entered. The figure is shown reduced from $8.5 x 11$ inches. 


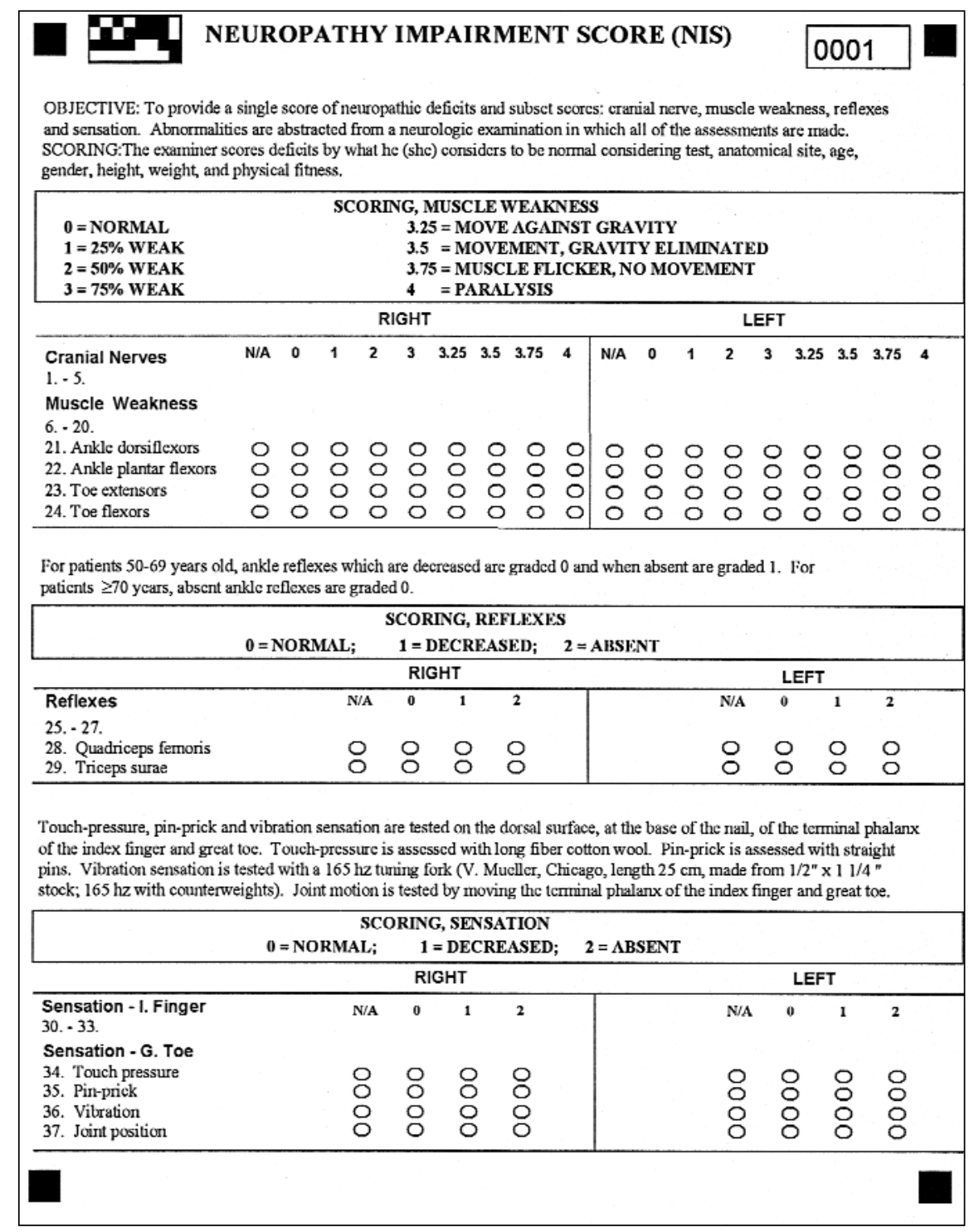

Figure 2: This is a composite of several parts of pages 4 and 5 of CNA (reduced in size) to provide information about Neuropathy Impairment Score (NIS). Provided is the layout used, the scoring of weakness, reflexes and sensation. Test items not shown in detail in this figure are: $1-5,6-20,25-27$, and 30-33.

(p 1), patient and study information (p 2, Figure 1), Lower Limb Function (LLF p 3), Neuropathy Impairment Score (NIS, p 4 to 5, Figure 2) and Neuropathy Symptoms and Change (NSC, p 7 to 12 , Figure 3 and 4). Patient, disease and demographic data are entered by indelible ballpoint pen darkening of appropriate blisters. The paper CNA form is completed in the office or at the bedside and may be evaluated by eye by the physician or preferably interactively by computer software (by scanner or by fax server, the latter being the preferred method). If evaluated at a central reading and quality assurance center (R+QA center), both the facsimile copy of CNA and the read-out of the scanned data are virtually immediately available for comparison. The form was prepared in English, but forms were also prepared in more than 10 other languages. For the latter, only the questions concerning symptoms were translated so that the wording about symptoms could be standard among languages. The translations were performed by professional translators and by neurologists whose native language was the language of the translation.

Each page of a CNA booklet has a checker-board identifier (upper left-hand corner Figure 1-4, so that the Teleform program recognizes only booklets belonging to a specific study), cornerstone symbols (in all four corners of each page of CNA, so that software can compensate for misalignment of pages with faxing), and a unique number (in the upper right-hand corner, for a specific CNA and a specific visit), which number is read by optical character recognition [OCR] and page number (also read 


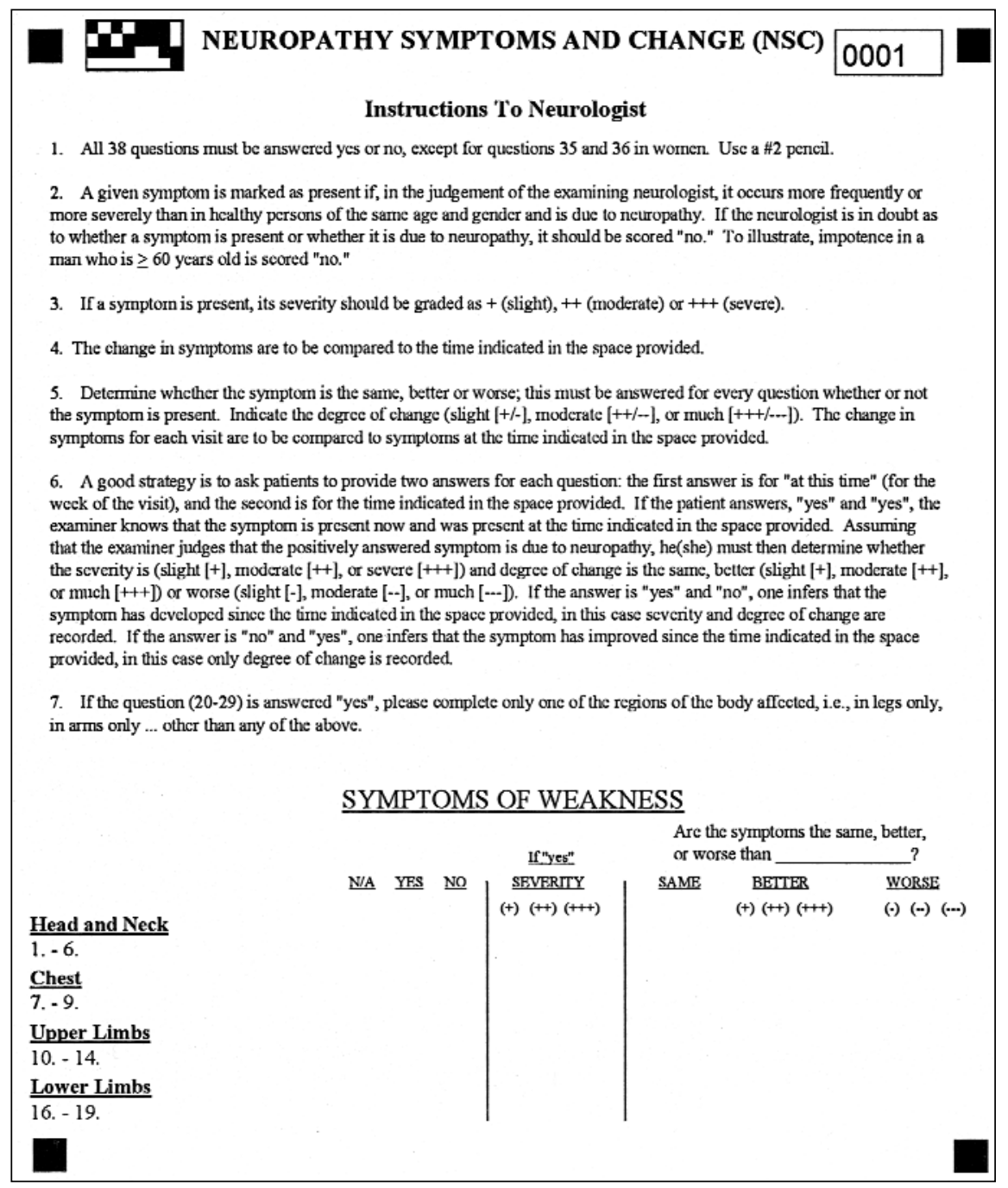

Figure 3: This is a composite of portions of page 6, 7 and 8 of CNA (reduced in size) to provide instructions about eliciting neuropathic symptoms and their scoring for the Neuropathy Symptoms and Change (NSC). Test items 1-19 are not provided in detail in this figure.

by OCR) to ensure that all pages and only the correct ones, are evaluated together.

Individual test items of CNA are scored separately for each side of the body, and include LLF, NIS, NSC, and disability (the scale determined by the investigator). The LLF tests the ability to walk on toes, on heels, and arise from a kneeled position. Each is scored as normal or abnormal. The scoring used for assessing neuropathic impairments are shown in Figure 2. The scoring of neuropathic symptoms is shown in Figure 3 and 4.

Clinical Neuropathy Assessment was designed to assess neuropathic impairments and symptoms for the entire body (e.g., for evaluation of such diseases as chronic inflammatory demyelinating polyradiculoneuropathy [CIDP]) or to assess only a limited region of the body. For CIDP, it is advantageous to assess all parts of the body, since all parts of the body may be affected, and to strike a balance between motor, sensory, and autonomic symptoms and impairments. For diabetic polyneuropathy, a more limited assessment (e.g., lower limbs only [LL]) might serve (e.g., NIS[LL] and NSC[LL]). For the latter purpose, the forms are printed so that anatomical regions not to be assessed have pre-filled blisters as showing no abnormality. This is a simpler and cheaper strategy than reprogramming a modified CNA form. Also, pre-filling non-needed blisters allows evaluation of a single limb or a single nerve.

\section{Computer software programs}

\section{CNA Data Entry}

This program ensures that all pages of CNA are entered for analysis, that blisters have been filled in for all queried items, and that the information comes from listed medical centers and investigators. Illogical answers are identified for correction (e.g., questions answered both "yes" and "no"). Also identified for correction in NSC is the response that a symptom is present, 


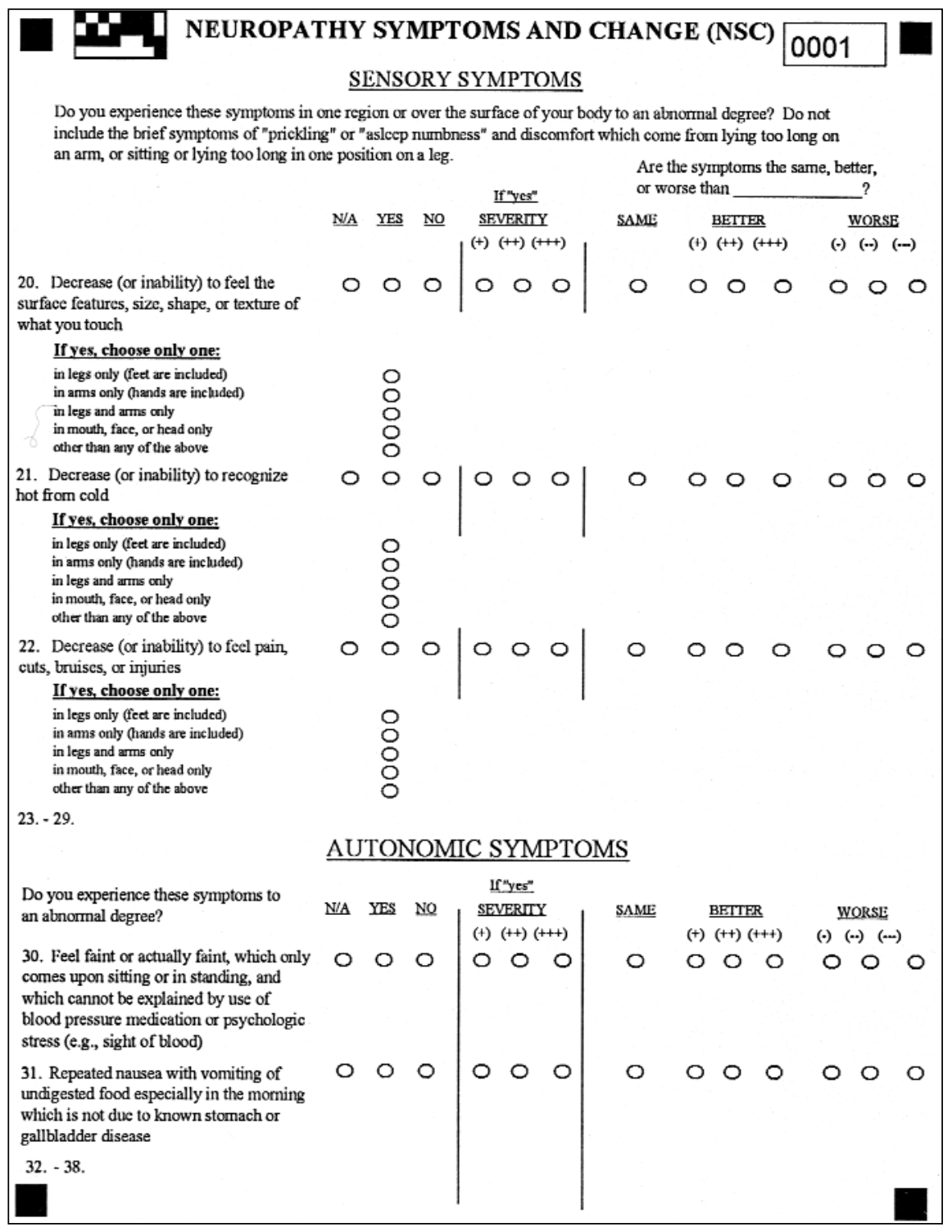

Figure 4: This is a composite of portions of page 9-12 of CNA (reduced in size) to provide a representative sample of questions from the Neuropathy Symptoms and Change (NSC) score. Test items 23-29 and 32-38 are not provided in the figure.

without indicating severity, or the anatomical location of the symptom. If a symptom is marked "no", severity should not be indicated but change must be shown (the symptom may have improved). If there are any incompletions or variances from a satisfactorily completed CNA, a message is automatically sent to the investigator listing the problem and asking for correction and resubmission.

\section{CNA Medical Evaluator}

After acceptance of the form by the CNA Data Entry program, CNA Medical Evaluator identifies divergences from the pre-determined template of characteristics of the neuropathy studied, identifies irregularities from the template of inclusion and exclusion criteria and discrepancies between dysfunctions, impairments, and symptoms. To illustrate, if a CNA of a young man of average weight is scored "abnormal" for walking on heels (item 2 of LLF) with ankle dorsiflexors scored 0 and 0 (item 21 of NIS, Box 2), it is declared discrepant by the program. Discrepancies must be corrected or explained to the R+QA center by the investigator. Personnel from the R+QA center are not permitted to make any judgements about patient or disease conditions - these must all come from participating investigators.

\section{CNA Checker Program}

This program lists all abnormalities (item-by-item) of dysfunctions, impairments, or symptoms from the database for a given CNA. The list can then be compared, line-by-line, against the facsimile of the image of the CNA booklet to ensure that all abnormalities of the paper facsimile of the source document 


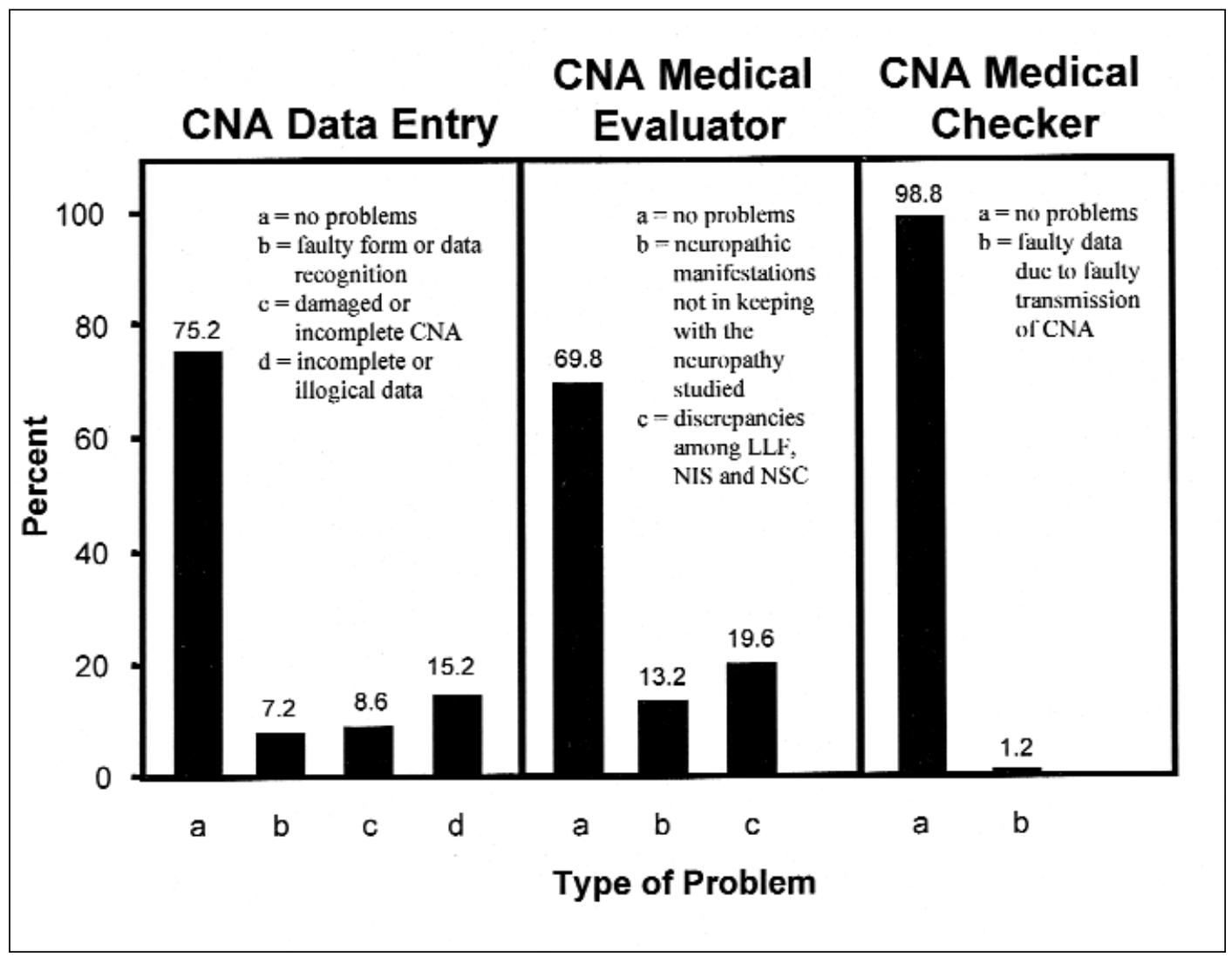

Figure 5: This figure provides a tally (percent) of problems which were identified (and then corrected) by using the interactive programmed evaluation of 500 CNAs in an Eli Lilly Inc. study of 500 consecutive submissions. The software program "Data Entry" identified almost 25\% of CNAs as having faulty form or data recognition, damaged or incompletions or incomplete or illogical data entry. The "Medical Evaluator" software program identified approximately $30 \%$ as showing either discrepancy from the medical template or discrepancies among test results. The final "Medical Checker" software program identified only 1.2\% of CNAs as having faulty data. In all cases, this was due to an easily recognizable line shift of data.

correspond to the ones that are in electronic memory. A dated and signed copy of the CNA (the source document) is retained at the investigator's center, and a facsimile of the CNA (and the paper record of all queries and responses) is kept in the R+QA center as evidence that the stored electronic data have been correctly entered into electronic memory and verified.

\section{Controlled clinical trials, epidemiologic surveys and use in medical practice}

Clinical neuropathy assessment was and is used in prospective, randomized, double-blind, placebo-controlled therapeutic trials in diabetic polyneuropathy of: 1) nerve growth factor (rhNGF, Genentech, Inc., San Francisco, CA and Roche Inc., Basel, Switzerland $>10,000$ examinations); 2) $\alpha$ lipoic acid (Asta Medica, Frankfurt, Germany $>2,000$ forms); and 3) Ly333531 (E. Lilly Inc., Indianapolis, IN, >1,000 forms). The form is also used for cross-sectional and longitudinal epidemiologic studies of healthy and diabetic patients in Rochester, MN and of Mdewakanton Dakota native people in Prairie Island, MN ( $>3,000$ forms). We also use CNA to periodically (every 3,6 and 12 months) evaluate patients with immune neuropathies to determine their need for beginning, changing, or stopping immunotherapy.

Tallying the frequency of problems and errors and time for analysis using CNA versus non-programmed evaluation

Of 500 serial CNAs in the E. Lilly Inc. study, we calculated the number and percent and type of problem which needed and was corrected using the three software programs written for this purpose.

In a second analysis, we compared the error rate and the time needed for the evaluation of 25 serial CNAs using scanning, and interactive programmed evaluation versus non-programmed evaluation without use of scannable records. In the first case (use of scannable CNAs and interactive programmed evaluation), all items showing abnormal values in database were checked against the original source document, line-by-line, to ensure accuracy of the data. In the second case (non-programmed evaluation), all checks of study and disease conditions and all information in the CNA record were doubly entered into computer memory. If any discrepancies were found between the doubly entered data, it was reconciled before the corrected data 
was re-entered. The database information of the $25 \mathrm{CNA}$ records obtained by the two approaches were then compared for accuracy.

\section{RESUlTS}

\section{General observations on use of CNA in controlled clinical trials}

A considerable effort and expense is needed to set up a scannable case report form such as CNA and the needed programs and systems for its evaluation, and this would probably not be justified unless the approach was needed for the evaluation of several thousand forms. The strategy of providing a comprehensive form, which could also be utilized for a more limited assessment by prefilling blisters of questions which do not need to be evaluated and so do not apply, allowed us to use the form for a variety of studies without need to reprogram CNA.

In international, multicenter trials of neuropathy treatment, CNA provided efficient, comprehensive, and reliable data collection, interactive analysis, and quality assurance with maintenance of an audit trail. Use of a scannable case report form (such as CNA) facilitates multi-user trials of therapy in diabetic neuropathy but is also usable for other neuropathies. Within minutes of completing CNA, it may be faxed (virtually instantaneously and from anywhere) to the R+QA center where the form can be immediately evaluated to ensure that it is correct, complete, and does not contain discrepant data. The original completed CNA form remains as the source document at the study center. At the R+QA center, the CNA is automatically received, night or day, and program evaluated to ensure that it comes from an approved study center, that it is complete, and without technical error and that study and disease criteria are met. If such criteria are not met, a message is automatically sent back to the investigator to correct omissions or errors and, under certain circumstances, to advise re-examination of the patient. Such automatic analysis and response is efficient and it was usual for us to respond within 24-72 hours. This rapid response made it possible to re-examine patients, if this was necessary, within the time frame of scheduled visits.

Rapid transmission of the facsimile of the case report form and interactive programmed analysis with maintenance of an audit trail made possible multicenter trials from different countries, rapid and efficient correction of problems near the time of a patient's visit, and a better surveillance of the disease and study conditions permitting patients to be included or excluded from study efficiently and providing frequent feedback interrogation of investigators' judgments if they were discrepant. With previous study approaches it may be weeks or months before the records are evaluated at a R+QA center, making it impossible to obtain valid re-assessments near the time of a patient's scheduled visits. At screening, patients could also be excluded from the study by the R+QA center.

\section{The nature and frequency of problems remedied by use of scannable CNA and interactive programmed evaluation}

From evaluation of 500 consecutive CNAs at our R+QA center in the Eli Lilly, Inc. study, approximately $25 \%$ of records needed corrections and resubmissions because of faulty submission, incomplete data or discrepant data. Generally these incompletions or problems were of single (or a few) items that were readily corrected, and the corrected CNA was accepted after resubmission. The Medical Evaluator Program identified $\sim 30 \%$ of CNAs as showing test characteristics outside of the predetermined disease or study criteria or discrepancies among test results. These problems were automatically sent to the investigator for a response. As a result, some patients were withdrawn from study, data were reconciled or were explained, and then overridden by the R+QA center. The Medical Checker Program identified only $1.2 \%$ of CNAs as having faulty entry of data. The abnormality was in all cases due to an abnormal line shift of one or two lines of the CNA so that the data fell into incorrect columns. This misalignment problem was readily recognized and corrected. A final line-by-line check of information in database against the facsimile of the original or corrected source document was possible so that complete and correct data could be analyzed.

\section{Comparison of the time and error rate of scanned and interactive programmed versus non-programmed evaluation of CNAs}

The performance of scannable case report forms and interactive programmed analysis was unequivocally superior to the same analysis using non-programmed evaluation of 25 consecutive CNAs evaluated for the Lilly, Inc. study both by the criteria of speed and accuracy. Using scannable records and interactive programmed analysis, complete evaluation was possible in $\sim 9$ minutes per CNA. The comparable time for the same analysis with non-programmed analysis was $\sim 50$ minutes. For the 25 CNAs evaluated, no errors were discovered between information in database as compared to a line-by-line comparison of the original or corrected facsimile using scannable records and interactive programmed evaluation. By comparison, non-programmed analysis and double keyboard entry disclosed discrepancies in 8 of 25 CNAs. After reconciliation of these errors, the data by programmed and nonprogrammed analysis were concordant.

\section{CNA in epidemiologic surveys}

The CNA form and the software and hardware needed for its evaluation did not need major alterations from what was used in multicenter trials. The advantages of using CNA is that standard data are entered only once and data are intensively and interactively evaluated for incompletions, discrepancies, and for accuracy.

\section{CNA in medical practice}

In our experience with several hundred CNAs in monitoring neuropathic status so as to begin, modify or stop immunotherapy in patients with immune neuropathies, we have found CNA to be valuable. It employs scales of impairment and symptoms, which are useful for initiating, changing or stopping immunotherapy. Additionally, an electronic record of dysfunctions, impairments, symptoms, disability, and of composite scores is maintained in database for later tracking of severity of neuropathy and for other analyses. We have used the standard tests of the CNA for monitoring immunotherapy treatment in chronic inflammatorydemyelinating polyradiculoneuropathy, multifocal motor neuropathy with conduction block, monoclonal gammopathies of undetermined significance neuropathy and for neoplastic and 
paraneoplastic neuropathies. Having a readily analyzable detailed electronic record of all serial examinations of a patient's neuropathy is useful not only for treatment decisions but also for later research needs.

\section{Discussion}

Scannable medical records whose information can be automatically read, evaluated, summarized, transferred and stored in database, are useful for a variety of medical purposes. The approach may be used as an update of a patient's medical, family, drug, treatment, physical fitness, life-style choices and risk-factor history prior to an event of clinical evaluation and care. ${ }^{1}$ More typically it may be used to develop a database useful in medical billing ${ }^{2}$ or for capture of data for later study of patient cohorts, disease classification, medical events, practices, treatments or outcomes. ${ }^{3-9}$ The approach has also been used to study environmental and occupational health issues ${ }^{10}$ or cancer prevalence and risk factors. ${ }^{11}$

Scannable case report forms and automatic reading, evaluation and transfer of information to database are also being introduced into pharmaceutical trials. At a former time, and even now, clinical report forms were developed for a specific trial. Data were manually entered, checked for completeness and errors at study sites and periodically by a visiting clinical research associate from the sponsoring company and then sent to the pharmaceutical house for manual double entry by keyboard and reconciliation of data and then entered into database. The use of scannable forms and the necessary hardware and computer software approaches have made it possible to create, modify, maintain, archive, retrieve, or transmit clinical data intended for submission to the Food and Drug Administration (FDA) (Guidance for Industry Computerized Systems Used in Clinical Trials, April 1999).

In the approach we developed and describe here, we use a standard paper form as the source document but all subsequent steps (reading the data by scanning, transference of the facsimile and of the data, interactive analysis, and entry into database) are automatic, but using interactive surveillance and quality control. We could have used a paperless computer approach for entry and further handling of data but decided against this approach because a paper source document was desired and because personal computers were not readily available or acceptable to all participating investigators. Also, the accuracy of video without a paper record by physicians has not been established.

Does the use of scannable case report forms (and the analysis by the computer programs described here) improve the accuracy of the information in database? Was time saved? Is the approach cost-effective? A difference in accuracy was observed between scanned and manual entry of information into database. With scanning of 500 serial CNA records, $1.2 \%$ of records were found to have faulty data entry, which in quality control was readily recognized as due to a line shift of the data, and then was readily corrected. For the 25 serial CNA evaluated in timed studies, no line shifts occurred. By comparison, when all steps of CNA data evaluation were done by hand and the data entered by keyboard, 8 of 25 records were found to have data entry discrepancies. When the differences between them were reconciled, the data was completely concordant with the scanned data entry results.
These results, therefore, indicate that scanned programmed assessment with adequate quality control tends to be more accurate, although both approaches give accurate final results. With respect to speed of assessment of case report forms, our results are different from what has previously been reported. Guerette et $\mathrm{al}^{5}$ compared the time needed and the error rate of data scanned or manually entered by keyboard into database and did not find a difference. These conclusions were supported by another study done by Nies et al. ${ }^{12}$ By contrast, we found a major savings in time (a five-fold improvement) by using scannable records and interactive programmed analysis. Strictly speaking, our analysis should not be compared to these earlier studies because more than accuracy of entered data by double keyboard entry versus scanning entry was involved in our comparison. We used CNA to monitor disease and study criteria and concordance of test data not compared in these earlier studies.

In summary, when there is a need to record, transfer and enter into database standard characterizing and clinical data on large numbers of patients, scannable records with interactive programmed analysis provide improved accuracy and speed and may have other advantages over normal double entry of data and may be cost effective.

\section{ACKNOWLEDGEMENTS}

We thank the physicians who have participated, or are participating in trials using CNA. The Genentech trial has been published and lists the names of co-investigators (Apfel SC, et $\mathrm{al}^{13}$ ). Jane Norell, B.S. and Carol Overland $(\mathrm{CO})$ performed the timed studies and Mary Lou Hunziker prepared the manuscript.

This work was supported in part from grants received from the National Institute of Neurologic Disorders and Stroke (NS36797). Financial support was also received from Genentech, Inc (San Francisco, CA), Roche, Inc. (Basel, Switzerland), Asta Medica, Inc. (Frankfurt, Germany), and Eli Lilly Inc. (Indianapolis, IN).

\section{REFERENCES}

1. Hagen PT, Turner D, Daniels L, Joyce D. Very large-scale distributed scanning solution for automated entry of patient information. Toward an electronic patient record '98: proceedings 1998; 1:228-232.

2. Adams WT, Snow GM, Helmick PM. Automated charge processing streamlines data entry. Health Financ Manage 2000; 54:50-53.

3. Shiffman RN, Brandt CA, Freeman BG. Transition to a computerbased record using scannable, structured encounter forms. Arch Pediatr Adolesc Med 1997; 151:1247-1253.

4. Allen DM, Hey LA, Heinz TR, Golal R, Levin LS. Development and implementation of an extremity free-tissue-transfer database. J Reconstr Microsurg 1997; 13:475-487.

5. Guerette P, Robinson B, Moran WP, et al. Teleform scannable data entry: an efficient method to update a community-based medical record? Community care coordination network Database Group. Proceedings - The Annual Symposium on Computer Applications in Medical Care 1995;86-90.

6. Moss RL, Kolaric D, Watts A. Therapeutic agents utilized in urban/rural prehospital care. Prehospital Disaster Med 1993; 8:161-164

7. Xakellis GC, Gjerde CL, Emerson M. Using an optical scanner and data base program to manage a family medicine teaching program. Fam Med 1994; 26:421-424.

8. McKegney FP, Schwartz CE, O’Dowd MA, Salamon I, Kennedy R. Development of an optically scanned consultation-liaison data base. Gen Hosp Psychiatry 1990; 12:71-76.

9. Dayhoff R, Kirin G, Pollock S, Todd S. Data capture workstations, scanned forms, and pen-based systems for clinician use. Medinfo 1995; 8(Pt 2): 1679 
10. O'Rourke MK, Fernandez LM, Bittel CN, et al. Mass data message: an automated data processing system used for NHEXAS, Arizona. National Human Exposure Assessment Survey. J Expo Anal Environ Epidemiol 1999; 9:471-484.

11. El Hattab O, Dayhoff RE. Automated medical records technology at the National Cancer Institute-Egypt: a case study in technology transfer. Medinfo 1995; 8(Pt 1):305-309.
12. Nies MA, Hein L. Teleform: a blessing or burden? Publ Health Nurs 2000; 17:143-145.

13. Apfel SC, Schwartz S, Adornato BT, et al. Efficacy and safety of recombinant human nerve growth factor in patients with diabetic polyneuropathy. A randomized controlled trial. JAMA 2000; 284:2215-2221. 